\title{
Diet, Gut Microbiota and COVID-19
}

\author{
Praveen Rishi ${ }^{1} \cdot$ Khemraj Thakur $^{1} \cdot$ Shania $_{\mathrm{Vij}^{1}}{ }^{1}$ Lavanya Rishi $^{2}$ • \\ Aagamjit Singh $^{3} \cdot$ Indu Pal Kaur $^{4}$ - Sanjay K. S. Patel ${ }^{5}$ Jung-Kul Lee ${ }^{5}$. \\ Vipin C. Kalia
}

Received: 28 July 2020/Accepted: 17 September 2020/Published online: 28 September 2020

(C) Association of Microbiologists of India 2020

\begin{abstract}
Worldwide, millions of individuals have been affected by the prevailing SARS-CoV-2. Therefore, a robust immune system remains indispensable, as an immunocompromised host status has proven to be fatal. In the absence of any specific antiviral drug/vaccine, COVID19 related drug repurposing along with various other nonpharmacological measures coupled with lockdown have been employed to combat this infection. In this context, a plant based rich fiber diet, which happens to be consumed by a majority of the Indian population, appears to be advantageous, as it replenishes the host gut microbiota with beneficial microbes thereby leading to a symbiotic association conferring various health benefits to the host including enhanced immunity. Further, implementation of the lockdown which has proven to be a good non-pharmacological measure, seems to have resulted in consumption of home cooked healthy diet, thereby enriching the beneficial microflora in the gut, which might have
\end{abstract}

Praveen Rishi

rishipraveen@yahoo.com; rishiparveen@pu.ac.in

$\bowtie$ Jung-Kul Lee

jkrhee@konkuk.ac.kr

$\triangle$ Vipin C. Kalia

vckaliaku@gmail.com

1 Department of Microbiology, Panjab University, BMS Block I, South Campus, Chandigarh, India

2 Panjab University Campus, Chandigarh, India

3 All India Institute of Medical Sciences (AIIMS), New Delhi, India

4 University Institute of Pharmaceutical Sciences, Panjab University, Chandigarh, India

5 Department of Chemical Engineering, Konkuk University, Seoul 05029, Republic of Korea resulted in better prognosis of COVID-19 patients in India in comparison to that observed in the western countries.

Keywords Association · COVID-19 · Diet · Dysbiosis · Gut microbiota $\cdot$ Symbiosis

\section{Introduction}

Ever since the outbreak of COVID-19 occurred in Wuhan, China in December 2019, it has rapidly spread throughout the globe. In view of the scourge of this disease world over, the World Health Organization (WHO) declared it as a pandemic on March 11, 2020 [1]. By now, it has affected approximately 16.5 million people worldwide (as on 28th July, 2020) and the number continues to increase. Thus, the entire human population is being threatened to get exposed to this highly contagious virus sooner or later [2]. Infection with COVID-19 has been associated with significant mortality, particularly in the high-risk group such as health care providers and elderly people, with or without comorbidities [3, 4]. Very recently, it has been proposed that people with an underlying chronic inflammation of the gut are more predisposed to a heightened cytokine storm when infected with this virus [5]. Cytokine storm has been defined as the over-production of early response cytokines leading to an increased risk of vascular hyper-permeability, multi-organ failure and eventually death, as a result of high cytokine concentrations which are not found to reduce over time [6].

Further, chronic gut inflammation has been suggested to result from a specific constitution of gut microbiome, with the latter being regulated by diet. For example, plant-based foods are likely to support a gut microbiome capable of inducing an appropriate level of anti-inflammatory 
response in the host in contrast to a pro-inflammatory immune response elicited by the gut microbiome of individuals consuming food products such as wheat, red meat and alcohol, thereby resulting in chronic gut inflammation [5]. Simultaneously, since many people had to undergo forced fasting during the COVID-19 pandemic, it might have affected their microbiota. The main hypothesis underlying the study is that, can this lead to susceptibility to infections? And can vegetarian diet help to boost immunity and help human beings to fight the pathogens? Believing the notion: 'We are, what we eat', this manuscript provides an opportunity to the researchers in the field of Microbiology and Medical health to carry out studies on the effect of forced fasting on gut microbiota and means to counter any potential ill effects. In this context, the present review envisages the possible association of diet and microbiota with the incidence/severity/recovery of COVID-19 cases during the lockdown period (from 25th March to 31st May 2020) in India.

\section{Diet and Gut Microbiota in the Population of Developed and Developing Countries}

Dietary habits and amount of food consumed has been reported to shape the microbiome [5]. Predominant microbial phyla of the human gastrointestinal tract include Bacteriodetes, Firmicutes, Actinobacteria, Fusobacteria, Proteobacteria and Verrucomicrobium [7]. Of these, Bacteriodetes (Bacteroides, Prevotella) and Firmicutes (Eubacterium, Lactobacillus) constitute more than $90 \%$ of the bacterial population inhabiting the colon. In early 1990s, Élie Metchnikoff reported that Bulgarians have very high life expectancy, which was attributed to the consumption of yoghurt, thereby meeting the requirement of harboring a good microbiota to maintain homeostasis [8]. Ever since then, several reports reflecting changes in the diet as well as the gut microbiota have made this hypothesis worth exploring. It is now a well-established fact, that modern Western diet consisting of food, which is processed and kept in cold storage, has less fiber content in comparison to the diets consumed by developing countries [9]. It has been shown experimentally that there was an increase in abundance of bacteria belonging to Firmicutes phylum with a simultaneous decrease in the bacteria of Bacteroidetes phylum in the gut of people residing in Western countries. Generally, Bacteriodetes are found to be dominant in people with vegetarian diet whereas Firmicutes are dominant in people having animal-based diet [10]. For example, it has been reported that children from Africa were enriched with Bacteroidetes due to high fiber content in their diet. Moreover, they possessed higher counts of bacteria, such as Prevotella and Xylanibacter, which have been reported to possess genes coding for molecules involved in hydrolysis of such complex plantbased polysaccharides (which are otherwise indigestible). They were also found to possess lower number of Firmicutes and demonstrated increased amount of short chain fatty acids (SCFAs) in their faeces as compared to the microbiota of the children of European origin [11]. The concentration of SCFAs is important at local (colon) or systemic (blood) levels for immunoregulation [12].

Even immigration to the US has been reported to westernize the human gut microbiome [13]. Migration, specially from a non-Western nation such as India to Western countries, has been reported to present with a decreased abundance of plant fibre degrading enzyme as a direct implication of decrease in the gut bacterial diversity. In these immigrants, Bacteroides strain has been found to displace Prevotella strains subject to the duration of stay in the USA. Moreover, loss in gut-microbial diversity has also been shown to increase with obesity and the problem stands exacerbated in future generations [14].

Bacterial genera such as Prevotella, Lactobacillus, and Carnobacterium were found to be more abundant in Indian population as compared to the Chinese population. Also, Prevotella and Bifidobacteria were the dominant genera in Indian population owing to the vegetarian diet followed by most inhabitants in contrast to Chinese inhabitants where Bifidobacteria and Blautia were found to be most abundant which can be attributed to the increased consumption of animal based diet therein [15]. Recently, Pareek et al. [16] compared the gut microbiota of Indian and Japanese population and have also indicated the diet dependent interaction between bacteria and fungi residing in the gut. It has been observed that presence of more plant polysaccharides in Indian diet as compared to that of Japanese leads to increased numbers of Prevotella and Candida in the gut of Indians. Moreover, Candida species were found to promote the proliferation of Prevotella when provided with arabinoxylan rich diet [16].

India records the second largest population along with being the seventh largest country in the world and exhibits diverse dietary habits, ethnicity, and lifestyle. Various studies have highlighted the link between the diet and gut microbiota profile of Indians [17, 18]. Core microbiota analysis of Indians revealed the distinctive predominance of genus Prevotella in $80 \%$ of the population while almost $55 \%$ of the population harbored Bacteroides, Prevotella, Megasphaera, and Roseburia in their gut [19]. One comparative study also indicated that Prevotella are the dominant species in the gut of most North-Central Indians, who mostly consume plant-based carbohydrate rich diet whereas bacteria such as Bacteroides, Fecalibacteria and Ruminococci make a significant part of gut microbiota of South Indians who mostly consume an omnivorous diet 
[18]. Another comparative study revealed that the gut bacterial profile of Indian tribal population is like that of the Mongolian population because of similar dietary habits [20]. The effect of urbanization on the gut microflora of humans in India has shown a clear difference between the gut microbiota profiles of the rural and urban counterparts [21]. It was observed that Bacteroides species were found to be abundant in Indians residing in urban areas whereas Prevotella species were dominant in rural population. Dietary habits have been reported as one of the driving factors for these differences.

\section{Dietary Nutrients}

Further, the amount of dietary nutrients including micronutrients (polyphenols and vitamins), macronutrients (carbohydrates, fats and proteins) as well as minerals and trace metals (magnesium, iron, selenium, zinc) have been reported to exhibit substantial effect on the gut microbiota [22]. In vitro studies have suggested that polyphenols such as phenols, flavonoids, and lignans present abundantly in raw vegetables and fruits along with cereals and drinks such as coffee, tea and wine could greatly modulate the gut flora favouring the growth of potentially beneficial organisms such as Lactobacillus, Bifidobacteria, Akkermansia and Fecalibacteria, simultaneously inhibiting the growth of potentially pathogenic bacteria such as Helicobacter pylori and Staphylococcus species [23]. Preclinical studies and clinical trials have shown that polyphenols affect the ratio of Firmicutes to Bacteroides (F/B) [24]. This could be attributed to the prebiotic like activity of the polyphenols (for example, inulin has been reported as a prebiotic for Lactobacillus plantarum [25] and their biotransformation to produce SCFAs, along with other bacterial metabolites, that contribute to the growth of beneficial bacteria resulting in a significant reduction in inflammation and thereby improving the systemic state of disease [26]. Likewise, vitamins C (lemon, spinach, broccoli), D (eggs, fish) and $\mathrm{E}$ (almonds, spinach, broccoli, olive oil) supplementation have been found to modulate health-beneficial microbiota (Bifidobacteria, Lactobacillus, and microbes of the genus Roseburia) and reduce the F/B ratio [27]. However, modulation of the gut microbial profile depends on the level of vitamins in the host. Therefore, clinical trial pertaining to concerns regarding excess vitamin supplementation are required to address these issues. Out of the minerals, zinc supplementation (milk products, nuts, read meat) has been reported to reduce the deleterious microbes and increase the beneficial ones in various preclinical studies [28].

Carbohydrates also play a pivotal role in influencing the gut flora. Plant derived carbohydrate based high-fiber diet was found to increase the abundance of Bifidobacterium and reduce the F/B ratio [29]. Most of the carbohydrates such as galacto-oligosaccharides, arabinoxylan, oligofructose institutes the growth of bacteria such as Bifidobacteria, Lactobacillus, Roseburia, etc., which have been known to confer health benefits and has been found to influence the F/B ratio [27]. Certain carbohydrates have been reported to possess restorative activity in reversing the state of dysbiosis, thereby indicating their potential as a possible therapeutic intervention for management of various metabolic diseases.

In case of fats, both the quantity as well as their type plays a major role in modulating the gut microbiota [27]. For example, saturated fat reduces beneficial microbes such as Bifidobacterium and Fecalibacterium, thereby increasing the $\mathrm{F} / \mathrm{B}$ ratio. On the contrary, unsaturated fat has been found to reduce detrimental microbes such as Escherichia and Streptococcus species and simultaneously increasing the beneficial bacteria such as Bifidobacteria and Akkermansia, hence lowering the F/B ratio. Moreover, studies have also reported that high fat deteriorates the density of beneficial microbes whereas following it with a low-fat diet reverses this effect. Similarly, based on their quality and quantity, proteins have also been reported to affect the heterogeneity and the constitution of gut flora [27]. For example, whey protein increases Bifidobacteria at low concentrations but decreases the same at higher concentrations. Furthermore, animal-based proteins (eggs, fish, meat) have been reported to increase deleterious gut microbiota, thereby increasing the susceptibility of an individual to intestinal inflammation. However, preclinical, and clinical trials are still being carried out to confirm these effects on gut microbiota. In view of the above-mentioned information, it is suggested that a healthy diet leads to symbiosis and contributes immensely in maintaining homeostasis. On the other hand, dysbiosis is linked to various kinds of gut inflammation which eventually culminate in gut-associated co-morbidities [30].

\section{Symbiosis, Immune Regulation, and Homeostasis}

Interdependence between the host and gut microbiota has been found to be indispensable [31]. Various studies have reported that mutualistic association between the two holds immense significance in maintaining an equipoise as the former provides the latter with a nutrient rich milieu and the latter in turn aids in various key functions necessary for host sustenance [32]. In this regard, the impact of gut microbiota can broadly be classified into structural, metabolic, and protective functions. The gut microflora has been known to strengthen the gut barrier by enhancing expression of tight junction proteins and simultaneously inducing IgA production and prominent bacteria that aid in both include Bacteroides spp., F. prausnitzii, Akkermansia 
muciniphila, Roseburia spp.; probiotic bacteria including Bifidobacterium and Lactobacillus spp. [33]. The metabolic functions include their ability to produce essential vitamins and simultaneously metabolize iron, other dietary carcinogens along with fermentation of non-digestible carbohydrates. The indigenous microflora also protects the host by preventing establishment of gut pathogens due to enhanced competitive binding by competing for nutrients, space, and host cell receptors along with production of various anti-microbial factors thereby resulting in pathogen displacement [7]. The fermentation of dietary fiber by gut microbes, resulting in formation of short chain fatty acids, has been further known to regulate immune functioning via various receptors and pathways involving molecules such as G-protein coupled receptors (GPCRs). GPCRs such as GPCR43 and GPCR120 have been reported to regulate anti-inflammatory pathways such as enhanced reactive oxygen species (ROS) mediated killing coupled with enhanced phagocytosis, induction of apoptosis, recruitment of specific proteins and cytokine production thereby contributing immensely to immune regulation [12]. All these findings substantiate the role of gut flora, its composition and its role in immune system regulation owing to its ability in producing molecules such as SCFA, peptidoglycans and polysaccharide A from a fibre rich diet, which significantly contributes to the maintenance of homeostasis. In addition to the regular diet intake, fasting is an integral part of many cultures worldwide and is considered auspicious traditionally. This concept has been getting scientific validations for its role in maintaining gut and immune homeostasis. Intermittent fasting (IF) (which includes alternate day fasting or time restricted feeding) has been reported to favorably influence the gut microbiota [34] by increasing the abundance of beneficial Akkermansia muciniphila and Bacteroides fragilis [35]. In fact, various animal trials and some human intervention studies have clearly demonstrated health benefits associated with IF in people with underlying diabetes, obesity, and cardiovascular ailments [36].

\section{Diet Induced Dysbiosis, Inflammation and Comorbidity}

It has been established that plant food-based diet promotes the microbes that ensue anti-inflammatory response and is thus, known to maintain a more diverse and stable microbiota [37]. In other words, a vegetarian diet seems to be more beneficial for human health due to its ability to maintain a state of symbiosis i.e., balanced microflora within the host. In contrast to this, certain food items such as gluten in wheat, red meat and alcohol can promote the growth of dysbiotic microorganisms which trigger a heightened pro-inflammatory response, thereby culminating in chronic inflammation [5]. This chronic inflammation has been reported to cause gut leakiness due to degradation of tight junctions by various proteases [38], thus allowing the dissemination of cells, pathogenic bacteria, and viruses into the blood circulation and transforming the existing state of gut inflammation into chronic systemic inflammation [39]. This chronic inflammation may remain undetected for years altogether, serving as a predisposing risk factor which may, at any time soon, aggravate into ailments and other serious manifestations, and therefore can be considered as a comorbidity. The term 'comorbidity' refers to any added illness in patients already suffering from a disease. In the recent decade, occurrence of psychiatric comorbidity in chronic disease has been widely observed and has been emphasized as an important aspect of disease management [40].

Various studies have reported the association of dysbiosis with the pathogenesis of various intestinal or extraintestinal disorders such as coeliac disease, cardiovascular disease, diabetes, and obesity [9, 41]. Similarly, Kedia et al. [7] have reported comparable changes in the gut microbiome of patients suffering from autoimmune (Inflammatory Bowel Disease, IBD) as well as infectious diseases, suggesting the role of inflammation in the altered status of the gut microbiome. Hence, it can also be inferred that comorbidity can arise because of dysbiotic inflammatory condition.

\section{Effect of Microbiota on COVID-19 Cases During Lockdown}

The prevailing COVID-19 pandemic situation arising from the spread of highly contagious virus led to a lockdown in various countries. Indian Government also announced the lockdown on March 25, 2020, further followed by three more lockdowns. Based on the interim guidelines of WHO, most of the countries opted for several non-pharmacological measures such as social distancing and masking along with lockdown of varying time intervals. Though a huge global economic loss was incurred due to enforcement of restriction on outdoor activities [42], but lockdown was propounded to be the most effective way to mitigate this dreadful situation in the absence of any treatment procedures or therapeutic/prophylactic agents. Lockdown implementation proved to be a reasonably effective strategy even in India (despite being one of the most populous country in the world), as by the end of 4th lockdown (31st May 2020), total number of confirmed infection cases in India were 182,143 (Fig. 1a) with 5164 deceased cases [43]. Thus, on an average, only 12 infected cases per lakh people were seen during the lockdown period (Fig. 1b). 
A

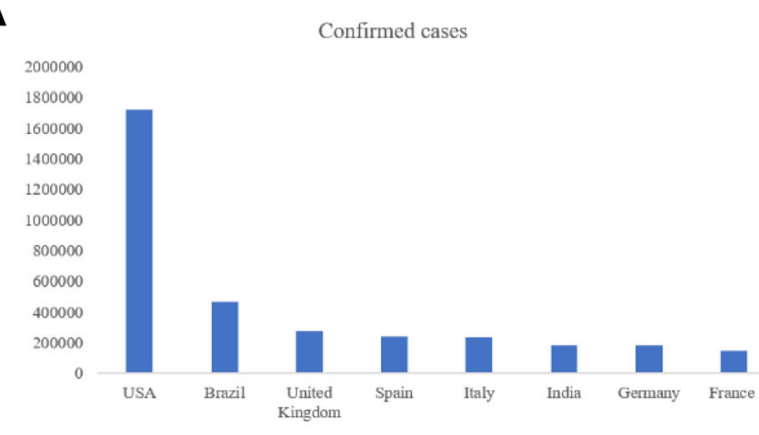

B

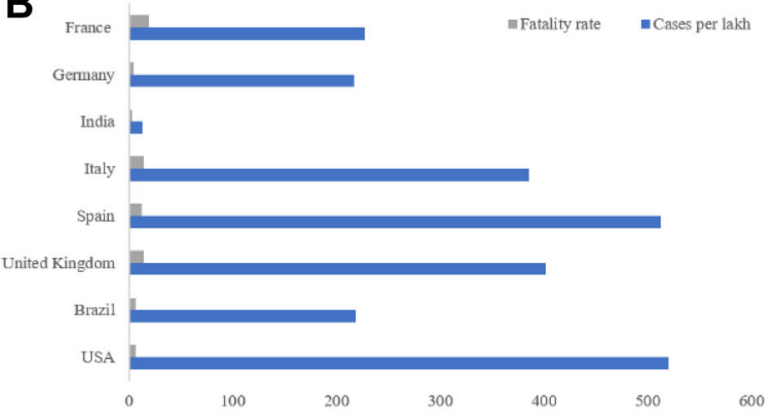

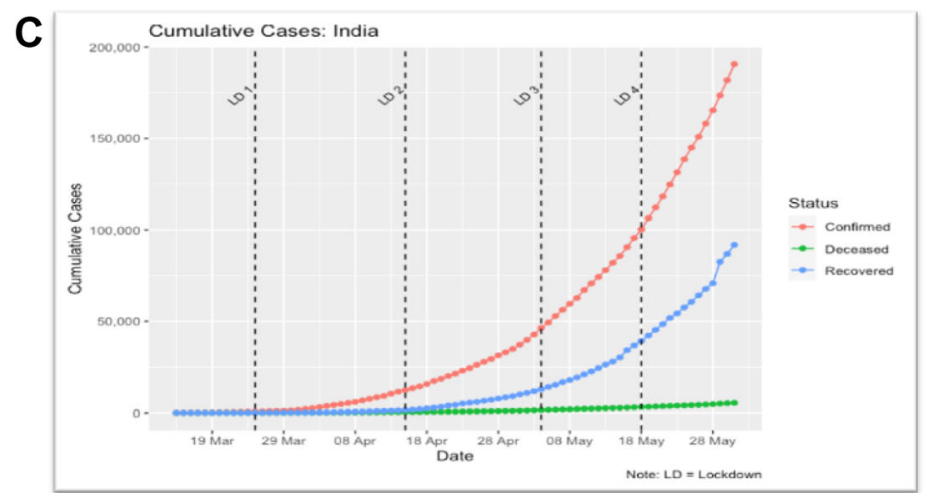

Fig. 1 a Confirmed COVID-19 cases in different countries on 31st May, 2020; b Comparative fatality rate of COVID-19 infection and cases per lakh population; c Cumulative cases (confirmed, recovered and deceased) in India during different phases of Lockdown. [Graph generated from the data retrieved from (http://www.covid19india.org) by using $\mathrm{R}$ script]
The fatality rate (2.83) of India was also observed to be the lowest as compared to the worst affected Western countries (Fig. 1b) and basic reproduction unit $\left(\mathrm{R}_{\mathrm{o}}\right)$ was significantly reduced during the course of lockdown from $R=1.26$ to $R=1.06$ till the end of 4th lockdown (31st May 2020). $R_{o}$ or basic reproduction unit is the ratio of transmission rate to recovery rate calculated by SIR modelling study [44] conducted in our lab (Data not reported here). A positive impact of lockdown up to the second lockdown period is clearly visible in Fig. 1c, after which a rapid upsurge in the cases was observed which can be mainly attributed to considerable relaxations made by the Government of India.

It may be argued that the number of confirmed cases represented may not be correct because of insufficient testing of the Indian population as only symptomatic people with a travel history to affected countries or people in close contact with the COVID-19 positive case were being tested [45]. In this case, it is also worth noting that in India, an enough percentage of infected individuals have been reported to be asymptomatic (approximately 80\%) [46]. Therefore, it can be presumed that even if infected cases are there, these are probably asymptomatic due to the healthy dietary habits and symbiosis. However, being asymptomatic is always a risk of transmission of infection to others and that is where measures like social distancing, masking and respiratory etiquettes prove to be of immense benefit.

Here, it may be inferred that plant based, home cooked, rich fiber diet consumed by the Indian population during lockdown, might have resulted in generation of symbiotic microflora, thereby eliciting anti-inflammatory responses. Indian diet is also rich in whole grains, which are a rich source of dietary fiber, carbohydrates, resistant starch and oligosaccharides. These are fermented in the colon after escaping digestion in the small intestine and result in production of SCFAs, which act as an energy source for the colonocytes, lower the colonic $\mathrm{pH}$ as well as alter the blood lipids, hence eliciting an immune response beyond the gut [47]. Whole grains are also rich in antioxidants such as phenolic compounds which have been shown to result in disease prevention [48]. Moreover, whole grains also possess certain compounds which may reduce the risk of chronic diseases such as diabetes, obesity, and cancer. Furthermore, state wise data distribution within India presented that the states where millet is the staple food, less prevalence of the disease was observed along with a higher recovery rate and a lower fatality rate. 
Simultaneously, a shift from animal-based diet to plantbased, fiber rich diet, has been reported to be associated with a change in microbiota (which changes within days to weeks) [49]. This shift was expected during the lockdown in the Indian population. This might have downregulated any pre-existing chronic inflammation, thereby reducing the occurrence of cytokine storm. Due to an almost total shutdown, it can be presumed that the public had limited access to animal based or ready to eat processed food, which is generally rich in refined carbohydrates and fats. The lockdown very successfully steered the public to consciously consuming homemade healthy diet, which probably resulted in reduced incidence/severity of disease during this period due to an improved microbiota or symbiosis.

It was also observed that besides having many cases, India was spared from the fatality caused by SARS-CoV-2 as compared to other countries, especially Western countries, such as USA (Fig. 1b). USA $(1,716,078)$ and Brazil $(465,166)$ have maximum number of cases followed by European countries [43]. Both the regions consume meat rich diet and saturated fatty acids. Pro-inflammatory responses triggered due to such a diet may lead to fatal complications in patients infected with COVID-19, where lung epithelial cell inflammation might have detrimental consequences. Moreover, it was also observed that due to lockdown restrictions in the Western countries, access to fresh vegetables or fruits were also restricted to the population, unlike India, where daily grocery items such as fresh fruits, vegetables, and milk were accessible to the public daily. The former situation might have resulted in increased consumption of processed food, leading to dysbiosis and simultaneously resulting in addition of more cytokines to the pro-inflammatory milieu. Increased consumption of processed foods which are mostly rich in fats, sugars, and salts, not only increases the risk of lifestyle diseases such as obesity and cardiovascular problems in patients suffering from chronic inflammation, but has also been found to have grave implications in enhancing COVID-19 related complications [47]. Most of the COVID-19 related deaths reported in India so far, might be attributed to the immunocompromised status of the individual as most of them were found to be suffering from some comorbidity such as hypertension, diabetes or other cardiovascular diseases [3].

Therefore, early implementation of lockdown by Indian Government was a great step towards preventing a catastrophe as there are over 8 crore hypertension patients and about 77 million diabetic patients in India [50]. A tool developed by the Oxford University, Oxford COVID-19 Government Response tracker (OFCGRT), also applauded the Indian Government for having done exceptionally well in curtailing the coronavirus outbreak [51].
An Italian Survey carried out by Di Renzo et al. [49], has also highlighted some of the positive effects of the lockdown, such as, a higher indulgence in physical activity and adoption of healthier lifestyles by quitting smoking. Moreover, an increased adherence to the Mediterranean diet was seen in the age group of 18-30, which might have reduced the risk of oxidative damage and in turn, the susceptibility to COVID-19 infection. This survey also emphasized the protective potential of healthy plant-based diet as compared to the processed food or animal-based diet.

Further during the lockdown period several of the daily wagers including migrant workers faced adversity and underwent phases of forced starvation. Similarly, people working in corporates away from their homes and taking their meals outside may also have faced situations where they underwent phases of no or little food during the lockdown. This situation is comparable to an intermittent fasting condition. In this context, it can be speculated that IF might have given the advantage to some by enriching their beneficial gut microbiota and thus the former nonpharmacological interventions can have special implication amidst the COVID-19 crisis, assisting the microbiomeimmune axis. It may be noted that perhaps, deaths which occurred amongst migrants were due to road accidents or starvation but not due to SARS-CoV-2 infection!

On the other hand, quarantine has also been reported to induce stress which leads to 'stress eating' wherein individuals tend to increasingly consume food with high fat and sugar content thereby leading to obesity. An obese individual has been reported to possess a decreased immune function demonstrated by a deficit in CD8+ T cells responses used to combat viral infections. This leads to higher mortality due to increased viral load in lungs and is also coupled with lung pathology [52]. Furthermore, in Western countries it was observed that a diet poor in fresh fruits and vegetables was more frequently ingested, thereby reducing the daily required intake of antioxidants and vitamins, which have been reported as potential weapons against the COVID-19. Therefore, strategies which encourage healthy diet and increased home-based physical activities should be implemented to reduce the risk of above-mentioned conditions.

In addition to a decline in the physical health of individuals due to ingestion of improper food, the effect of quarantine in deteriorating the mental health, especially in children, was also observed [53]. Herein, we would like to point out that such a condition may be avoided if the person is accustomed to healthy diet or diet supplemented with healthy microbes such as probiotics [54, 55]. Disturbances at the gut-brain axis, occurring due to various gastro-intestinal conditions, have been implicated in various mental health abnormalities as well such as anxiety, mood 
disorders and depression [56]. Probiotics have been reported to restore this balance and act as 'psychobiotics', thereby serving as an alternate treatment option. In this context, we recently studied the effect of a potential probiotic strain, Lactiplantibacillus plantarum (RTA 8), on psychological, histological and physiological status of diseased host and its role in ameliorating host health by modulating various aspects of the gut-brain axis [57]. The schematic representation of the possible association as mentioned above has been shown in Fig. 2. Given the purported association of microbiota with COVID-19 cases, studies on analysis of gut microbiome using faecal samples of these cases are warranted to validate this hypothesis.

Briefly, it can be concluded that the high fatality rate in Western countries as compared to India, during the lockdown, can be attributed to (1) their animal based diet which increases dysbiosis, hence increasing the pro-inflammatory cytokines resulting in cytokine storm in contrast to the Sattvic diet of the Indian culture including plant based diet and use of traditional medicine as recommended by the Ministry of AYUSH (Ayurveda, Yoga and Naturopathy, Unani, Siddha and Homeopathy), Government of India [58]; (2) the unhealthy dietary habits during lockdown, especially attributed to limited access to fresh grocery shopping, thus resulting in an increase in underlying conditions; (3) the late implementation of lockdown in contrast to the timely implementation of lockdown by the
Government of India; (4) the possibility of a more virulent variant of COVID-19 strain prevalent in Western countries in comparison to that prevalent in India due to high mutation rate in the genome of SARS-CoV-2 virus $[59,60]$ and; (5) India, being a developing country, is more exposed to infections as compared to the developed nations, thereby resulting in priming of their immune response against a wide variety of microbes leading to a better immune status than that observed in the Western population.

\section{Concluding Remarks and Future Perspectives}

There has been a growing appreciation of the role played by commensal microbiota in human health. Here, diet has been found to play a crucial role in modulating the gut microbiota, thereby resulting in symbiosis or dysbiosis. In this context, people should be enlightened about the importance of daily intake of a balanced diet which can be better defined and prescribed through the help of nutritional biologists and food industries. A balanced diet, like the ones prepared by the dieticians in hospitals and hostels of educational institutions, can also be defined for public. The same can also be supplied to the migrants and the economically weaker sections of the society to prevent malnutrition. Furthermore, the importance of intermittent fasting or following a fasting-mimicking diet can be an

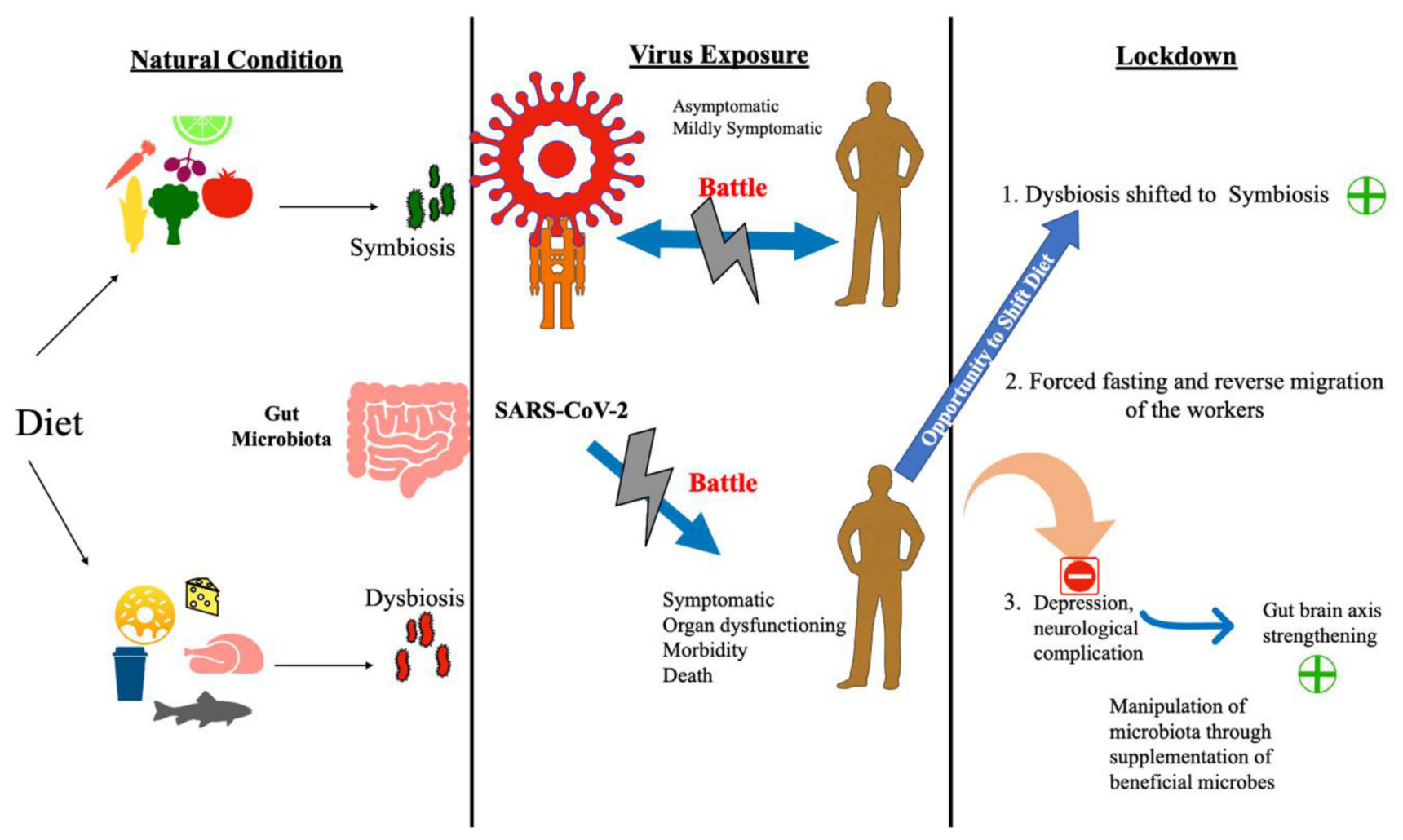

Fig. 2 Schematic representation of possible association of diet derived microbiota with susceptibility to/clinical outcome of COVID-19 
important advisory to the population, for not only restoring beneficial gut microbiota but promoting overall health as well.

Rapid advancements in the molecular techniques for assessment of human gut microbiome has helped in defining the diversity and metabolic properties of the gut microbiota. Intestinal microbiota of the host can be modulated through administration of synbiotics (probiotics and prebiotics), thereby conferring holistic health benefits. Developing such a combination of probiotics and prebiotics may ultimately prove to be more cost effective than developing new drugs. Gut microbiota can be modulated by enriching it with beneficial ones and eliminating the pathogenic microbes using antipathogens as an alternative to antibiotics [61-67]. The acceptance of scientific community/physicians for their application, specific to the control of infection, beyond the accepted source of health promoting effects is important. In addition to the regulatory and scientific constraints, public acceptance may be another hurdle which needs to be surmounted by educating and enhancing awareness to increase intake of probiotics/ prebiotics along with plant-based fiber rich diet to the recommended level.

As per the experience gathered during the lockdown period, a significant increase in the cleanliness quotient has been observed in the environment which is crucial for decreasing the incidence of infections along with contributing to robust health status of individuals. As environment is directly related to health, therefore, even post this COVID-19 pandemic, lockdown for a specific period once in every year may be practiced enriching the environment as well as health [68]. This would also give opportunity, particularly to the next generation, in inculcating good habits of healthy diet to strengthen their immune system.

In this regard, the concept 'Let food be thy medicine', proposed by Hippocrates way back in $431 \mathrm{BC}$, needs to be revisited, particularly during such situation as the present one, when there is no therapeutic or protective agent available and for now, cure lies only in prevention.

Acknowledgements This work was supported by DST-PURSE grant provided to Panjab University (PR) and Brain Pool Grant (NRF2020H1D3A2A01060467) by National Research Foundation of Korea (NRF) to work at Konkuk University (VCK). This research was also supported by Basic Science Research Program through the NRF (NRF-2019R1C1C1009766) funded by the Ministry of Science, ICT and Future Planning (SKSP).

\section{References}

1. Mitra P, Misra S, Sharma P (2020) COVID-19 pandemic in India: what lies ahead. Indian J Clin Biochem 35:257-259. https://doi. org/10.1007/s12291-020-00886-6
2. Raje DV, Bajaj A, Chakraborty M, Purohit HJ (2020) Cumulative active and recovery rates based criterion for gradual lockdown exit: a global observation of SARS COV-2 management. medRxiv. https://doi.org/10.1101/2020.06.05.20123364

3. Dey JK, Dey SK (2020) SARS-CoV-2 pandemic, COVID-19 case fatality rates and deaths per million population in India. J Bioinform Comput Syst Biol 2:110

4. Sood U, Gupta V, Kumar R, Lal S, Fawcett D, Rattan S, Poinern GEJ, Lal R (2020) Chicken gut microbiome and human health: past scenarios, current perspectives, and futuristic applications. Indian J Microbiol 60:2-11. https://doi.org/10.1007/s12088-01900785-2

5. Luthra-Guptasarma M, Guptasarma P (2020) Inflammation begets hyper-inflammation in: diet-derived chronic inflammation promotes runaway acute inflammation resulting in cytokine storms. Res Gate. https://doi.org/10.13140/RG.2.2.17723.44323

6. Jose RJ, Manuel A (2020) COVID-19 cytokine storm: the interplay between inflammation and coagulation. Lancet Respir Med 8:E46-E47. https://doi.org/10.1016/S2213-2600(20)30216-2

7. Kedia S, Rampal R, Paul J, Ahuja V (2016) Gut microbiome diversity in acute infective and chronic inflammatory gastrointestinal diseases in North India. J Gastroenterol 51:660-671. https://doi.org/10.1007/s00535-016-1193-1

8. Mackowiak PA (2013) Recycling Metchnikoff: probiotics, the intestinal microbiome and the quest for long life. Front Public Health 1:52. https://doi.org/10.3389/fpubh.2013.00052

9. Narayanan S, Pitchumoni CS (2020) Dietary fiber. In: Pitchumoni C, Dharmarajan T (eds) Geriatric gastroenterology. Springer, Cham, pp. 1-16. https://doi.org/10.1007/978-3-319-90761-1_271

10. Senghor B, Sokhna C, Ruimy R, Lagier JC (2018) Gut microbiota diversity according to dietary habits and geographical provenance. Hum Microbiome J 7:1-9. https://doi.org/10.1016/j. humic.2018.01.001

11. De Filippo C, Di Paola M, Ramazzotti M, Albanese D, Pieraccini G, Banci E, Miglietta F, Cavalieri D, Lionetti P (2017) Diet, environments, and gut microbiota. A preliminary investigation in children living in rural and urban Burkina Faso and Italy. Front Microbiol 8:1979. https://doi.org/10.3389/fmicb.2017.01979

12. Maslowski KM, Mackay CR (2011) Diet, gut microbiota and immune responses. Nat Immunol 12:5-9. https://doi.org/10.1038/ ni0111-5

13. Vangay P, Johnson AJ, Ward TL, Al-Ghalith GA, Shields-Cutler RR, Hillmann BM, Lucas SK, Beura LK, Thompson EA, Till LM, Batres R (2018) US immigration westernizes the human gut microbiome. Cell 175:962-972. https://doi.org/10.1016/j.cell. 2018.10.029

14. Davis CD (2016) The gut microbiome and its role in obesity. Nutr Today 51:167-174. https://doi.org/10.1097/NT. 0000000000000167

15. Jain A, Li XH, Chen WN (2018) Similarities and differences in gut microbiome composition correlate with dietary patterns of Indian and Chinese adults. AMB Express 8:1-12. https://doi.org/ 10.1186/s13568-018-0632-1

16. Pareek S, Kurakawa T, Das B, Motooka D, Nakaya S, RongsenChandola T, Goyal N, Kayama H, Dodd D, Okumura R, Maeda Y, Fujimoto K, Nii T, Ogawa T, Lida T, Bhandari N, Kida T, Nakamura S, Nair GB, Takeda K (2019) Comparison of Japanese and Indian intestinal microbiota shows diet-dependent interaction between bacteria and fungi. NPJ Biofilms Microbiomes 5:37. https://doi.org/10.1038/s41522-019-0110-9

17. Tandon D, Haque MM, Shaikh S, Dubey AK, Mande SS (2018) A snapshot of gut microbiota of an adult urban population from Western region of India. PLoS ONE 13:e0195643. https://doi.org/ 10.1371/journal.pone.0195643 
18. Dhakan DB, Maji A, Sharma AK, Saxena R, Pulikkan J, Grace T, Gomez A, Scaria J, Amato KR, Sharma VK (2019) The unique composition of Indian gut microbiome, gene catalogue, and associated fecal metabolome deciphered using multi-omics approaches. Gigascience 8:giz004. https://doi.org/10.1093/giga science/giz004

19. Kulkarni AS, Kumbhare SV, Dhotre DP, Shouche YS (2019) Mining the core gut microbiome from a sample Indian population. Indian J Microbiol 59:90-95. https://doi.org/10.1007/ s12088-018-0742-0

20. Dehingia M, Talukdar NC, Talukdar R, Reddy N, Mande SS, Deka M, Khan MR (2015) Gut bacterial diversity of the tribes of India and comparison with the worldwide data. Sci Rep 5:18563. https://doi.org/10.1038/srep18563

21. Monaghan T, Sloan TJ, Stockdale SR, Blanchard AM, Emes RD, Wilcox M, Biswas R, Nashine R, Manke S, Gandhi J, Jain P (2019) Metagenomics reveals impact of urbanisation in central India on the human gut microbiome and its antimicrobial resistance profiles. Res Sq. https://doi.org/10.21203/rs.2.17205/v1

22. Leeming ER, Johnson AJ, Spector TD, Le Roy CI (2019) Effect of diet on the gut microbiota: rethinking intervention duration. Nutrients 11:2862. https://doi.org/10.3390/nu11122862

23. Williamson $G$ (2017) The role of polyphenols in modern nutrition. Nutr Bull 42:226-235. https://doi.org/10.1111/nbu.12278

24. Corrêa TA, Rogero MM, Hassimotto NM, Lajolo FM (2019) The two-way polyphenols-microbiota interactions and their effects on obesity and related metabolic diseases. Front Nutr 6:188. https:// doi.org/10.3389/fnut.2019.00188

25. Rishi P, Arora S, Kaur UJ, Chopra K, Kaur IP (2017) Better management of alcohol liver disease using a 'microstructured synbox' system comprising L. plantarum and EGCG. PLoS ONE 12:e0168459. https://doi.org/10.1371/journal.pone.0168459

26. Filosa S, Di Meo F, Crispi S (2018) Polyphenols-gut microbiota interplay and brain neuromodulation. Neural Regen Res 13:2055-2059. https://doi.org/10.4103/1673-5374.241429

27. Yang Q, Liang Q, Balakrishnan B, Belobrajdic DP, Feng QJ, Zhang W (2020) Role of dietary nutrients in the modulation of gut microbiota: a narrative review. Nutrients 2:381. https://doi. org/10.3390/nu12020381

28. Zackular JP, Moore JL, Jordan AT, Juttukonda LJ, Noto MJ, Nicholson MR, Crews JD, Semler MW, Zhang Y, Ware LB, Washington MK (2016) Dietary zinc alters the microbiota and decreases resistance to Clostridium difficile infection. Nat Med 22:1330-1334. https://doi.org/10.1038/nm.4174

29. Avila-Nava A, Noriega LG, Tovar AR, Granados O, Perez-Cruz C, Pedraza-Chaverri J, Torres N (2017) Food combination based on a pre-hispanic Mexican diet decreases metabolic and cognitive abnormalities and gut microbiota dysbiosis caused by a sucroseenriched high-fat diet in rats. Mol Nutr Food Res 61:1501023. https://doi.org/10.1002/mnfr.201501023

30. Viana SD, Nunes S, Reis F (2020) ACE2 imbalance as a key player for the poor outcomes in COVID-19 patients with agerelated comorbidities-role of gut microbiota dysbiosis. Ageing Res Rev. https://doi.org/10.1016/j.arr.2020.101123

31. Gaur M, Vasudeva A, Singh A, Sharma V, Khurana H, Negi RK, Lee J-K, Kalia VC, Misra R, Singh Y (2019) Comparison of DNA extraction methods for optimal recovery of metagenomic DNA from human and environmental samples. Indian J Microbiol 59:482-489. https://doi.org/10.1007/s12088-019-00832-y

32. Lepage $P$ (2017) The human gut microbiota: Interactions with the host and dysfunctions. Rev Mal Respir 34:1085-1090. https://doi. org/10.1016/j.rmr.2016.11.003

33. Wells JM, Brummer RJ, Derrien M, MacDonald TT, Troost F, Cani PD, Theodorou V, Dekker J, Méheust A, De Vos WM, Mercenier A (2017) Homeostasis of the gut barrier and potential biomarkers. Am J Physiol Gastrointest Liver Physiol 312:G171G193. https://doi.org/10.1152/ajpgi.00048.2015

34. Li L, Su Y, Li F, Wang Y, Ma Z, Li Z, Su J (2020) The effects of daily fasting hours on shaping gut microbiota in mice. BMC Microbiol 20:1-8. https://doi.org/10.1186/s12866-020-01754-2

35. Özkul C, Yalınay M, Karakan T (2019) Islamic fasting leads to an increased abundance of Akkermansia muciniphila and Bacteroides fragilis group: a preliminary study on intermittent fasting. Turk J Gastroenterol 30:1030. https://doi.org/10.5152/tjg. 2019.19185

36. Patterson RE, Laughlin GA, LaCroix AZ, Hartman SJ, Natarajan L, Senger CM, Martínez ME, Villaseñor A, Sears DD, Marinac CR, Gallo LC (2015) Intermittent fasting and human metabolic health. J Acad Nutr Diet 115:1203-1212

37. Tomova A, Bukovsky I, Rembert E, Yonas W, Alwarith J, Barnard ND, Kahleova H (2019) The effects of vegetarian and vegan diets on gut microbiota. Front Nutr 6:47. https://doi.org/10.3389/ fnut.2019.00047

38. Van Spaendonk H, Ceuleers H, Witters L, Patteet E, Joossens J, Augustyns K, Lambeir AM, De Meester I, De Man JG, De Winter BY (2017) Regulation of intestinal permeability: the role of proteases. World J Gastroenterol 23:2106-2123. https://doi.org/ 10.3748/wjg.v23.i12.2106

39. Yu LC (2018) Microbiota dysbiosis and barrier dysfunction in inflammatory bowel disease and colorectal cancers: exploring a common ground hypothesis. J Biomed Sci 25:1-4. https://doi.org/ 10.1186/s12929-018-0483-8

40. Jones R (2010) Chronic disease and comorbidity. Br J Gen Pract 60:394-394. https://doi.org/10.3399/bjgp10X502056

41. Singhvi N, Gupta V, Gaur M, Sharma V, Puri A, Singh Y, Dubey GP, Lal R (2020) Interplay of human gut microbiome in health and wellness. Indian J Microbiol 60:26-36. https://doi.org/10. 1007/s12088-019-00825-x

42. Mehta K, Jha SS (2020) COVID-19: a nightmare for the Indian economy. UGC Care J. https://doi.org/10.2139/ssrn.3612676

43. World Health Organization (2020) Coronavirus disease (COVID19) situation report-132, vol $31-5$

44. Reluga TC (2010) Game theory of social distancing in response to an epidemic. PLoS Comput Biol 6:1-9. https://doi.org/10. 1371/journal.pcbi.1000793

45. Varghese GM, John R (2020) COVID-19 in India: Moving from containment to mitigation. Indian J Med Res 151:136-139. https://doi.org/10.4103/ijmr.IJMR_860_20

46. Gupta N, Agrawal S, Ish P, Mishra S, Gaind R, Usha G, Singh B, Sen MK (2020) Safdarjung Hospital COVID 2019 working group. Clinical and epidemiologic profile of the initial COVID-19 patients at a tertiary care centre in India. Monaldi Arch Chest Dis 90:1294

47. Den Besten G, van Eunen K, Groen AK, Venema K, Reijngoud DJ, Bakker BM (2013) The role of short-chain fatty acids in the interplay between diet, gut microbiota, and host energy metabolism. J Lipid Res 54:2325-2340. https://doi.org/10.1194/jlr. R036012

48. Lee JK, Patel SK, Sung BH, Kalia VC (2020) Biomolecules from municipal and food industry wastes: an overview. Bioresour Technol 298:122346. https://doi.org/10.1016/j.biortech.2019. 122346

49. Di Renzo L, Gualtieri P, Pivari F, Soldati L, Attinà A, Cinelli G, Leggeri C, Caparello G, Barrea L, Scerbo F, Esposito E (2020) Eating habits and lifestyle changes during COVID-19 lockdown: an Italian survey. J Transl Med 18:229. https://doi.org/10.1186/ s12967-020-02399-5

50. IDF (2019) Diabetes atlas, 9th edn. International Diabetes Federation, Brussels, Belgium. https://www.diabetesatlas.org 
51. University of Oxford (2020) Corona virus Government response tracker. Available: https://www.bsg.ox.ac.uk/research/researchprojects/oxfordcovid-19-government-response-tracker

52. Mattioli AV, Sciomer S, Cocchi C, Maffei S, Gallina S (2020) Quarantine during COVID-19 outbreak: changes in diet and physical activity increase the risk of cardiovascular disease. Nutr Metab Cardiovasc Dis. https://doi.org/10.1016/j.numecd.2020.05. 020

53. Thakur K, Kumar N, Sharma N (2020) Effect of the pandemic and lockdown on mental health of children. Indian J Pediatr 87:552. https://doi.org/10.1007/s12098-020-03308-w

54. Kumar R, Sood U, Gupta V, Singh M, Scaria J, Lal R (2020a) Recent advancements in the development of modern probiotics for restoring human gut microbiome dysbiosis. Indian J Microbiol 60:12-25. https://doi.org/10.1007/s12088-019-00808-y

55. Naumova N, Alikina T, Tupikin A, Kalmykova A, Soldatova G, Vlassov V, Kabilov M (2020) Human gut microbiome response to short-term Bifidobacterium-based probiotic treatment. Indian $\mathbf{J}$ Microbiol. https://doi.org/10.1007/s12088-020-00888-1

56. Verma H, Phian S, Lakra P, Kaur J, Subudhi S, lal R, Dogra Rawat C (2020) Human gut microbiota and mental health: advancements and challenges in microbe-based therapeutic interventions. Indian J Microbiol. https://doi.org/10.1007/s12088020-00898-Z

57. Kaur A, Chopra K, Kaur IP, Rishi P (2020) Salmonella strain specificity determines post-typhoid CNS complications: intervention by Lactiplantibacillus plantarum at gut-brain axis. Front Microbiol 11:1568. https://doi.org/10.3389/fmicb.2020.01568

58. Patel SK, Lee JK, Kalia VC (2020) Deploying biomolecules as anti-COVID-19 agents. Indian J Microbiol 60:263-268. https:// doi.org/10.1007/s12088-020-00893-4

59. Bajaj A, Purohit HJ (2020) Understanding SARS-CoV-2: genetic diversity, transmission and cure in human. Indian $\mathrm{J}$ Microbiol 60:398-401. https://doi.org/10.1007/s12088-020-00869-4

60. Kumar R, Verma H, Singhvi N, Sood U, Gupta V, Singh M, Kumari R, Hira P, Nagar S, Talwar C, Nayyar N, Anand S, Rawat DC, Verma M, Negi RK, Singh Y, Lal R (2020) Comparative genomic analysis of rapidly evolving SARS CoV-2 viruses reveal mosaic pattern of phylogeographical distribution. mSystems. https://doi.org/10.1128/mSystems.00505-20

61. Kalia VC, Purohit HJ (2011) Quenching the quorum sensing system: potential antibacterial drug targets. Crit Rev Microbiol 37:121-140. https://doi.org/10.3109/1040841X.2010.532479

62. Kalia VC, Raju SC, Purohit HJ (2011) Genomic analysis reveals versatile organisms for quorum quenching enzymes: acyl-homoserine lactone-acylase and-lactonase. Open Microbiol J 5:1-11. https://doi.org/10.2174/1874285801105010001

63. Kalia VC (2013) Quorum sensing inhibitors: an overview. Biotechnol Adv 31:224-245. https://doi.org/10.1016/j.bio techadv.2012.10.004

64. Kumar P, Patel SKS, Lee JK, Kalia VC (2013) Extending the limits of Bacillus for novel biotechnological applications. Biotechnol Adv 31:1543-1561. https://doi.org/10.1016/j.bio techadv.2013.08.007

65. Kalia VC, Wood TK, Kumar P (2014) Evolution of resistance to quorum-sensing inhibitors. Microb Ecol 68:13-23. https://doi. org/10.1007/s00248-013-0316-y

66. Kalia VC, Patel SKS, Kang YC, Lee J-K (2019) Quorum sensing inhibitors as antipathogens: biotechnological applications. Biotechnol Adv 37:68-90. https://doi.org/10.1016/j.biotechadv. 2018.11.006

67. Parasuraman P, Devadatha B, Sarma VV, Ranganathan S, Ampasala DR, Reddy D, Kumavath R, Kim I-W, Patel SKS, Kalia VC, Lee J-K, Siddhardha B (2020) Inhibition of microbial quorum sensing mediated virulence factors by Pestalotiopsis sydowiana. J Microbiol Biotechnol 30:571-582. https://doi.org/ 10.4014/jmb.1907.07030

68. Gupta V, Kumar R, Sood U, Singhvi N (2020) Reconciling hygiene and cleanliness: a new perspective from human microbiome. Indian J Microbiol 60:37-44. https://doi.org/10.1007/ s12088-019-00839-5

Publisher's Note Springer Nature remains neutral with regard to jurisdictional claims in published maps and institutional affiliations. 